\title{
Acute sleep deprivation induces cardioprotection against ischemia/ reperfusion injury through reducing inflammatory responses: the role of central GABA-A receptors
}

\author{
Hoda Parsa ${ }^{1}$, Mahdieh Faghihi ${ }^{1}$, Gholam A. Kardar ${ }^{3}$ and Alireza Imani ${ }^{1,2}$ \\ ${ }^{1}$ Department of Physiology, School of Medicine, Tehran University of Medical Sciences, Tehran, Iran \\ ${ }^{2}$ Occupational Sleep Research Center, Baharloo Hospital, Tehran University of Medical Sciences, Tehran, Iran \\ ${ }^{3}$ Immunology, Asthma \& Allergy Research Institute, Children Medical Center, Tehran University of Medical Sciences, Tehran, \\ Iran
}

\begin{abstract}
Sleep is considered as a physiological regulator in the body. Gamma-aminobutyric acid (GABA) is a neurotransmitter that modulates sleep and affects cardiac functions. We evaluated effects of acute sleep deprivation (SD) on cardiac hemodynamic parameters, expression of pro-inflammatory cytokines, and Heat shock protein (Hsp70), serum level of lactate dehydrogenase (LDH) and prooxidant/antioxidant balance (PAB). Male Wistar rats were bilaterally cannulated in the central nucleus of amygdala (CeA) and saline or bicuculline was injected 24 hours prior to induction of 30 minute ischemia following 120 minute reperfusion. Forty-eight animals were randomly divided into four groups: Control (CONT), bicuculline (BIC), acute SD and bicuculline + acute sleep deprivation (BIC+SD). Animals in SD and BIC+SD groups were put in an aquarium for inducing sleep deprivation. SD attenuated LDH, pro-inflammatory cytokines and PAB; improved cardiac hemodynamic parameters and increased $H s p 70$ in non-infarcted area as compared to CONT. Administration of bicuculline increased LDH, pro-inflammatory cytokines and PAB, reduced cardiac hemodynamic parameters and $H s p 70$ as compared to CONT. Furthermore, bicuculline administration prior to acute sleep induction decreased SD effects on LDH, PAB, Hsp 70, cardiac hemodynamic parameters and pro-inflammatory cytokines. Induction of SD prior to ischemia/reperfusion induces cardioprotection through suppressing inflammatory responses.
\end{abstract}

Key words: Acute sleep deprivation - Cardioprotection - GABA - Inflammatory responses Lactate dehydrogenase

\begin{abstract}
Abbreviations: cDNA, complementary DNA; CeA, central nucleus of amygdala; GABA, gammaaminobutyric acid; HPRT, hypoxanthine phosphoribosyl transferase-encoding gene; Hsp, heat shock protein; IL, interleukin; IR, ischemia-reperfusion; LDH, lactate dehydrogenase; LVEDP, left ventricular end diastolic pressure; LVSP, left ventricular systolic pressure; MI, myocardial infarction; MIRI, myocardial IR injury; NF- $\mathrm{kB}$, nuclear factor- $\mathrm{kB}$; nqRT-PCR, quantitative reverse transcription polymerase chain reaction; NTS, nucleus tractus solitarius; PAB, prooxidant/ antioxidant balance; PCR, polymerase chain reaction; ROS, reactive oxygen species; SD, sleep deprivation; TNF- $\alpha$, tumor necrosis factor $\alpha$; UPS, ubiquitin proteasome system.
\end{abstract}

Correspondence to: Alireza Imani, Department of Physiology, School of Medicine, Tehran University of Medical Sciences, Tehran, Iran

E-mail: aimani@tums.ac.ir

\section{Introduction}

Cardiac reperfusion after an acute myocardial infarction (MI) often results in death of previously viable myocytes, a phenomenon termed as "Myocardial IR injury" (MIRI). 
Subsequent to necrotic cardiomyocyte death, an inflammatory cascade is activated and led to cardiac tissue damage (Ling et al. 2013). Sleep is a modulator of behaviour and physiological responses of the body and therefore, the duration and the quality of sleep can affect functioning of many organs. In this aspect, it has been reported that acute sleep deprivation shows protective effects (Edalatyzadeh et al. 2016; Pace et al. 2017). In the bidirectional crosstalk between sleep and the immune system, cytokines as 'sleep regulatory substance' play a prominent role in the homeostatic regulation of sleep in normal physiological conditions. However, experimental induction of sleep loss does not seem to alter circulating markers of inflammation (Dimitrov et al. 2015; Irwin et al. 2016). Gamma amino butyric acid (GABA) is a well-known regulatory neurotransmitter, its receptors are widely distributed in central nucleus of the amygdala (CeA) which modulates sleep (Liu et al. 2009; Chanana et al. 2016). GABA modulates cardiovascular functions by affecting central nervous system through nucleus tractus solitarius (NTS) (Avolio et al. 2014) and peripheral tissues. GABA has been shown to alter vascular tone and sympathetic activity in various tissue/organs like artery, kidney, mesenteric arterial bed, and the pulmonary artery in rabbit, rat and cat respectively (Lingeshwar et al. 2016). Evidence suggests that proinflammatory molecules released during autoimmune encephalomyelitis may affect central GABA activity (Sallam et al. 2016).

One of the central players in inflammatory signaling is the transcription factor nuclear factor $-\kappa \mathrm{B}(\mathrm{NF}-\kappa \mathrm{B})$. Numerous reports have shown that NF- $\kappa \mathrm{B}$ is activated after myocardial ischemia-reperfusion (IR) (Ling et al. 2013). NF- $\kappa \mathrm{B}$ translocate to the nucleus after releasing from its

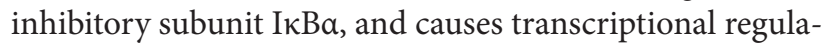
tion of the expression of interleukins and cytokines. The $\mathrm{NF}-\kappa \mathrm{B}-$ mediated inflammatory cascade can be perpetuated through the ability of NF- $\kappa \mathrm{B}$ targets, such as tumor necrosis factor $\alpha$ (TNF- $\alpha$ ) and interleukins to further activate NF- $\kappa B$ signaling, as well as by the infiltration of neutrophils and other mononuclear inflammatory cells at sites of injury (Ling et al. 2013). Lactate dehydrogenase (LDH) is a marker of myocardial injury which increases after IR (Cheng et al. 2016). It has been shown that LDH level increases in cardiac tissue after chronic sleep deprivation (Jeddi et al. 2016). Several recent studies have suggested the role of exaggerated reactive oxygen species (ROS) production and suppressed endogenous antioxidant defense such as heat shock proteins (Hsp's) in the pathogenesis of myocardial IR injury (Rani et al. 2013). Hsp 70 inhibits cardiomyocyte necroptosis through repressing autophagy in myocardial IR injury (Rani et al. 2013). Additionally, it has been reported that Hsp70 levels alters during sleep deprivation (Lapshina et al. 2010). The expression of $H s p 70$ has been shown to markedly increase in patients with sleep apnea (Lavie et al. 2010).
Although many studies have evaluated the role of inflammation in mediating IR injury, but the cardio protective role of acute sleep deprivation (SD) in suppression of IR-induced inflammation has not yet been studied.

\section{Material and Methods}

\section{Animals}

Forty-eight male adult Wistar rats (250-300 g) were housed in the animal house of the physiology department providing $12 \mathrm{~h} \mathrm{light/dark}$ cycle, temperature $23 \pm 2^{\circ} \mathrm{C}$, and unlimited access to food and water. The experimental protocols (Fig. 1) in this study were conformed to the Guidelines of the Care and Use of Laboratory Animals published by National Institutes of Health (NIH Publication No.85-23, revised 1996) and was further approved by the institutional ethical committee of Tehran University of Medical Sciences (Tehran, Iran). Acute sleep deprivasion was induced from 7:00 AM for $24 \mathrm{~h}$.

\section{Groups}

The central nucleus of amygdala (CeA) was bilaterally cannulated in all animals. After 5 days recovery, saline or bicuculline (a GABA-A antagonist) was injected into CeA. $24 \mathrm{~h}$ after saline or bicuculline injection, all rats were underwent $30 \mathrm{~min}$ ischemia followed by $120 \mathrm{~min}$ reperfusion for induction of IR. Animals were randomly divided into four groups (animal/group $n=12$ ) as below: (1) Control (CONT) group: saline was injected and $24 \mathrm{~h}$ later underwent for $30 \mathrm{~min}$ ischemia followed by $120 \mathrm{~min}$ reperfusion, (2) Bicuculline (BIC) group : bicuculline as GABA-A antagonist was administrated $24 \mathrm{~h}$ prior to $30 \mathrm{~min}$ ischemia followed by 120 min reperfusion, (3) Acute sleep deprived (SD) group : after administration of saline, animals were put in the aquarium by small plates for $24 \mathrm{~h}$ induction of acute sleep deprivation, and then subjected to $30 \mathrm{~min}$ ischemia followed by 120 min reperfusion, (4) Bicuculline + acute sleep deprivation $(\mathrm{BIC}+\mathrm{SD})$ group : bicuculline was injected Intra-CeA, then animals were put in aquarium by small plates for $24 \mathrm{~h}$ to induce acute sleep deprivation, then underwent for $30 \mathrm{~min}$ ischemia followed by $120 \mathrm{~min}$ reperfusion. At the end of reperfusion, blood samples were collected from anesthetic rat's heart. Then hearts were separated for genetic assessment.

\section{Stereotaxic surgery and drugs}

All rats were anesthetized by ketamine $(50 \mathrm{mg} / \mathrm{kg})$ and $\mathrm{xy}-$ lazine ( $5 \mathrm{mg} / \mathrm{kg}, i . p$.$) , and then were placed in a stereotaxic$ frame (Stoelting, USA) and the stainless steel guide cannulas (23 gauge, $15 \mathrm{~mm}$ length) were bilaterally implanted into the CeA with following coordinates according to Paxinus 


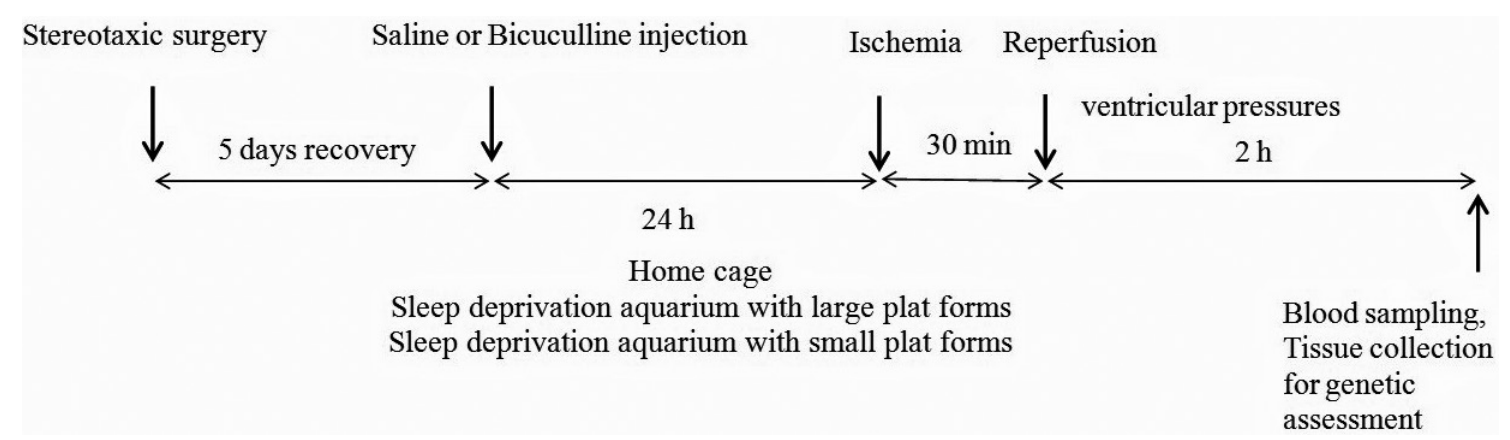

Figure 1. The experimental protocol.

and Whatson atlas (Anterior-Posterior: -2.8 , Lateral: \pm 4.6 , Dorsal-Ventral: -8.1) (Paxinos et al. 2007; Riahi et al. 2013).

Bicuculline methiodide (Sigma Aldrich) $0.1 \mathrm{nmol} / 0.5 \mu \mathrm{l}$ dissolved in normal saline. Although bicuculline methiodide is use for epilepsy induction, but this dosage that we administrated is not epileptic (Winn et al. 1980; Abbas 2015). Saline or bicuculline was injected $0.5 \mu \mathrm{l}$ at each side intra-CeA.

\section{Acute sleep deprivation induction}

24-h sleep deprivation was induced by putting the animals in an 8 circular small platforms aquarium $(125 \times 44 \times 44 \mathrm{~cm}$ and $6.5 \mathrm{~cm}$ in diameter) has been explained before (Parsa et al. 2016; Zagaar et al. 2016). The EEG recording confirmed the induction of sleep deprivation by multiple plate forms model (Arthaud et al. 2015).

\section{Myocardial ischemia and reperfusion}

All rats were anesthetized with thiopental $(60 \mathrm{mg} / \mathrm{kg}$, i.p. $)$ and the left anterior descending coronary artery (LAD) was ligated from origin according to our previous studies (Imani et al. 2011; Azizi et al. 2015).

\section{Blood sampling and Elisa assessment}

Blood samples were collected from the heart at the end of experiment for biochemical analysis (LDH, TNF- $a$ and prooxidant/antioxidant balance (PAB)). The samples were centrifuged at $7,000 \mathrm{rpm}, 4^{\circ} \mathrm{C}$, for $20 \mathrm{~min}$; the serum was removed and stored at $-70^{\circ} \mathrm{C}$ until biochemical analysis. Serum level of LDH, TNF- $a$, and PAB was measured by a commercial ELISA kit (Pars azmoon, Iran), according to the manufacturer's instructions.

\section{Assessment of myocardial function}

Changes of heart function were assessed by measuring of cardiac hemodynamic parameters. A small incision was made to the right of the midline in the neck. The right common carotid artery was exposed and cannulated with a PE50 catheter connected to the Power lab system through pressure transducer. Catheter was pushed down until it had reached the left ventricular lumen. Left ventricular systolic pressure (LVSP) and left ventricular end diastolic pressure (LVEDP) were all monitored and recorded by Powerlab data acquisition system (AD Instrument, Australia) (Azizi et al. 2015).

\section{Real-time polymerase chain reaction (Real-Time PCR)}

At the end of study, we removed the myocardial samples ( $n$ $=4$ ), rinsed in PBS, frizzed in liquid nitrogen and stored at $-80^{\circ} \mathrm{C}$. Total RNA was extracted from frozen infarcted and non-infarcted area by using Trizol (Invitrogen, Carlsbad, CA). A PrimeScript RT reagent kit (Takara, Cat. RR037A) was used to provide complementary DNA (cDNA) as templates for quantitative reverse transcription polymerase chain reaction (qRT-PCR), for quantification of gene expression, we used Rotor-Gene 6000 (Qiagen). Real-Time PCR analysis was provided by the use of SYBER Permix Ex Taq (Takar, Cat.RR280L). The value for each sample was an average of two independent PCR measurements. Hypoxanthine phosphoribosyl transferase-encoding gene (HPRT) was used as normalized to control (Azizi et al. 2015). The specific primer sequences are listed in Table 1.

\section{Statistical analysis}

Statistical analyses were done by one way ANOVA followed by Tukey post hoc test, using SPSS software (Version 22, SPSS IBM, Chicago, IL). All data are shown as mean \pm standard error of the mean (SEM) and $p<0.05$ was considered statistically significant. Sample size was determined based on previous studies which used to obtain significant results (Azizi et al. 2013, 2015). None of the samples were excluded from the analysis. The animals were randomly allocated to experimental groups and no blinding was observed during the assessment of experimental outcomes. 
Table1. The sequence of primers used in Real-Time PCR

\begin{tabular}{|c|c|c|}
\hline Gene name & Primer sequence & PCR product size \\
\hline Hsp70 & $\begin{array}{l}\text { R: 5'- TGAAGACCAGCACCGTGGA -3' } \\
\text { F: 5'- TGGCGTCATTCCGTTCCTTC -3' }\end{array}$ & 138 \\
\hline$I L-6$ & $\begin{array}{l}\text { R: 5'- AGCCAGAGTCATTCAGAGCAA-3' } \\
\text { F: 5'- TTGGTCCTTAGCCACTCCTTC -3' }\end{array}$ & 150 \\
\hline$T N F \alpha$ & $\begin{array}{l}\text { R: 5'-ACCACGCTCTTCTGTCTACTGA-3' } \\
\text { F: 5'-GGCTTGTCACTCGAGTTTTGAGA -3' }\end{array}$ & 85 \\
\hline HPRT & $\begin{array}{l}\text { R: 5'-GCAGGTCAGCAAAGAACTTATAGCC-3' } \\
\text { F: 5'-CTCATGGACTGATTATGGACAGGAC-3' }\end{array}$ & 123 \\
\hline
\end{tabular}

\section{Results}

\section{Effects of acute sleep deprivation on LDH level in serum}

$\mathrm{LDH}$ was measured as a marker of myocardial injury. Our results showed that bicuculline administration in BIC group increased LDH level (5901.8 $\pm 487.8,4016.8 \pm 264.7$; $p<0.01)$ as compared to CONT group. Acute sleep deprivation in SD group decreased LDH level (2601.8 $\pm 367.2 ; p<$ 0.05 ) as compared to CONT group. Bicuculline injection prior acute sleep deprivation in $\mathrm{BIC}+\mathrm{SD}$ group increased $\mathrm{LDH}$ in serum $(4490 \pm 225.2, p<0.01)$ as compared to SD group (Fig. 2).

\section{Effects of acute sleep deprivation on PAB level in serum}

Results shown in Fig. 3, explain that bicuculline administration in BIC group increased PAB level as compared to

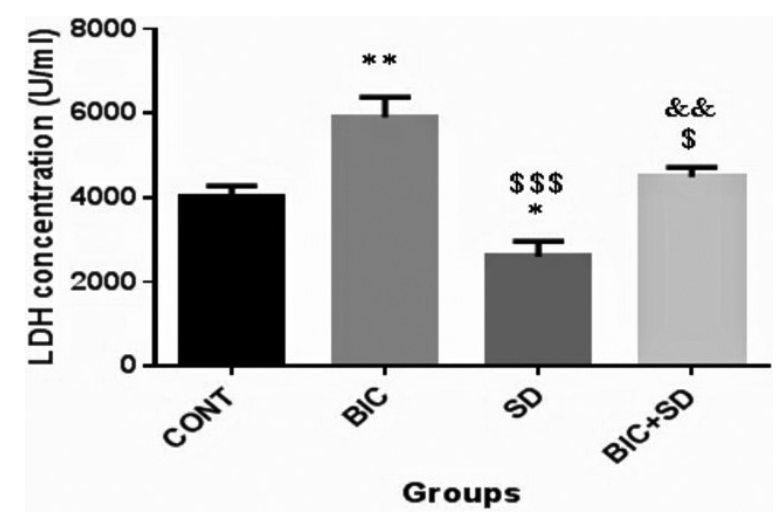

Figure 2. Lactate dehydrogenase (LDH) concentration in serum. All samples were analyzed in duplicate. Data are presented as mean \pm SEM (animals/group $n=6$ ). Groups: BIC, bicuculline; BIC+SD, bicuculline + acute sleep deprivation; CONT, ischemia-reperfusion; SD, acute sleep deprivation. ${ }^{\star} p<0.05$ and ${ }^{* *} p<0.01 v s$. CONT group, ${ }^{\$} p<0.05$ and ${ }^{\$ \$ \$} p<0.001 v s$. BIC group, ${ }^{\& \&} p<0.01 v s$. SD group.
CONT group $(13.41 \pm 2.31,2.67 \pm 0.64 ; p<0.01)$. Acute sleep deprivation in SD group decreased $\mathrm{PAB}$ level none significantly as compared to CONT group (1.94 \pm 0.34$)$. Bicuculline injection prior acute sleep deprivation in $\mathrm{BIC}+\mathrm{SD}$ group increased PAB level in serum none significantly as compared to SD group $(4.35 \pm 0.35)$.

\section{Assessment of cardiac hemodynamic parameters}

To assessment the myocardial hemodynamic parameters, we evaluated left ventricular systolic and end diastolic pressure. Our results showed that administration of bicuculline in BIC group decreased LVSP $(81 \pm 3.5)$ and increased LVEDP $(6.5 \pm 0.3)$ as compared to CONT group (LVSP: $100.4 \pm 1.6$, LVEDP: $4 \pm 0.6, p<0.05)$. Acute sleep deprivation induction in SD group increased LVSP $(121.3 \pm 1.6)$ and declined LVEDP $(1.3 \pm 0.5)$ as compared to CONT (LVSP: $p<0.05$, LVEDP: $p<0.01$ ) and BIC group (LVSP: $p<0.001$, LVEDP: $p<0.01)$. Injection of bicuculline prior to acute sleep dep-

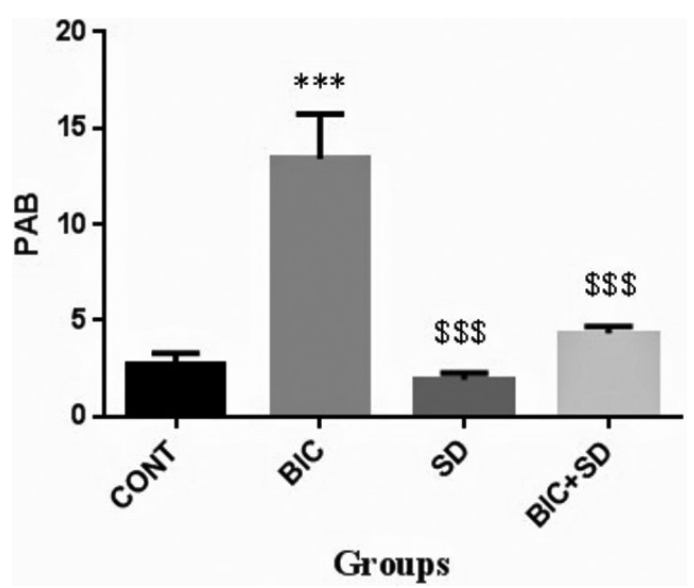

Figure 3. PAB concentration in serum. All samples were analyzed in duplicate. Data are presented as mean \pm SEM (animals/group $n=6) .{ }^{* *} p<0.01 v s$. CONT group, $\$ \$ \$ p<0.001 v s$. BIC group. $\mathrm{PAB}$, prooxidant/antioxidant balance. For more abbreviations, see Fig. 2. 
rivation in $\mathrm{BIC}+\mathrm{SD}$ group decreased LVSP $(102.7 \pm 6)$ and increased LVEDP $(3.6 \pm 055)$ as compared to SD group $(p<$ 0.05) (Fig. 4).

\section{Effects of acute sleep deprivation on gene expression}

To evaluate the effect of acute sleep deprivation on gene expression (Hsp70, IL-6, TNF- $\alpha$ ) in heart tissue, we divided left ventricle into infarcted and non-infarcted area in all groups.

\section{Effects of acute sleep deprivation on Hsp70 expression}

Fig. 5A shows Hsp70 expression in infarcted and noninfarcted area. Our results declare that $H s p 70$ expression was decreased fallowing bicuculline injection in BIC group $(0.9 \pm 0.01, p<0.001)$ as compared to CONT group. Acute sleep deprivation in SD group increased Hsp70 expression $(1.44 \pm 0.02, p<0.001)$ as compared to BIC group. induction of acute sleep deprivation after bicuculline administration in $\mathrm{BIC}+\mathrm{SD}$ group increased $H s p 70$ expression in infarcted area $(1.15 \pm 0.06, p<0.001)$ as compared to BIC group.

Hsp70 expression is declined in non-infarcted area in BIC group $(0.02 \pm 0.01, p<0.001)$ as compared to CONT group. Induction of acute sleep deprivation in SD group increased $H s p 70$ expression $(70 \pm 0.26, p<0.001)$ in noninfarcted area as compared to CONT group. Injection of bicuculline prior to acute sleep deprivation in $\mathrm{BIC}+\mathrm{SD}$ group decreased elevated $H s p 70$ expression induced by acute sleep deprivation in non-infarcted area $(1.2 \pm 0.24$, $p<0.001)$ as compared to BIC group.

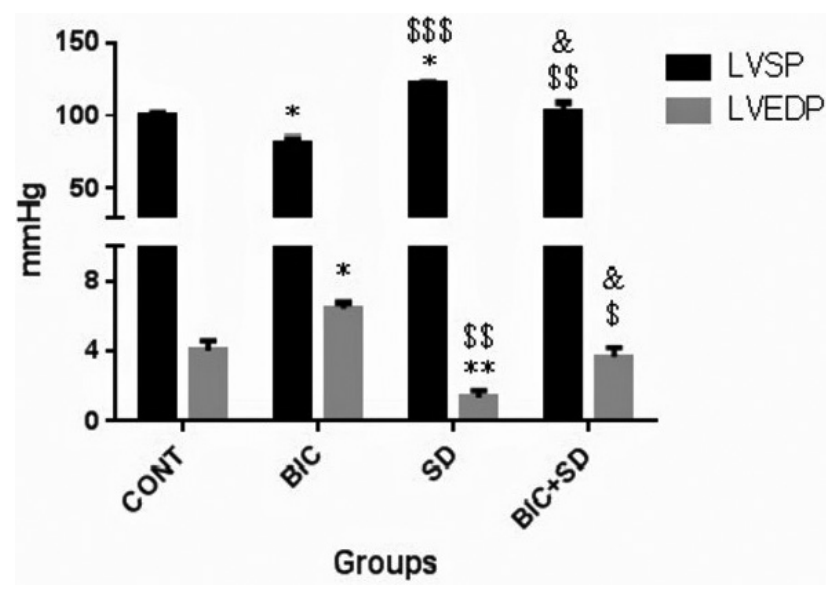

Figure 4. Left ventricular systolic pressure (LVSP) and left ventricular end diastolic pressure (LVEDP). ${ }^{*} p<0.05$ and ${ }^{* *} p<0.01$ vs. CONT group, ${ }^{\$} p<0.05,{ }^{\$} p<0.01$ and ${ }^{\$ \$} p<0.001 v s$. BIC group, ${ }^{\&} p<0.05$ vs. SD group. For more abbreviations, see Fig. 2.


Figure 5. Fold of changes of Hsp70 (A), IL-6 (B) and TNF- $\alpha$ (C) mRNA expression vs. CONT group in infarcted area and non-infarcted area. All samples were analyzed in duplicate. Data are presented as mean \pm SEM (animals/group $n=5$ ). ${ }^{* *} p<$ 0.01 and ${ }^{* * *} p<0.001$ vs. CONT group, ${ }^{\$ \$} p<0.001$ vs. BIC group, ${ }^{\& \& \&} p<0.001$ vs. SD group. Hsp, heat shock protein; IL, interleukin; TNF- $\alpha$, tumor necrosis factor $\alpha$. For more abbreviations, see Fig. 2. 


\section{Effects of acute sleep deprivation on IL-6 expression}

As seen in Fig. 5B, $I L-6$ expression in infarcted area of BIC group increased $(4.45 \pm 0.34, p<0.001)$ as compared to CONT group. IL-6 expression in SD group is decreased ( 0.7 $\pm 0.02, p<0.001)$ as compared to CONT group. Injection of bicuculline into central nucleus of amygdala before acute sleep deprivation induction in BIC+SD group increased $I L-6$ expression $(1.7 \pm 0.3, p<0.001)$ as compared to SD group. Il-6 expression in non-infarcted area increased in BIC group $(2.5 \pm 0.4, p<0.001)$ as compared to CONT group. Acute sleep deprivation in SD group decreased $I L-6$ expression in non-infarcted area $(0.2 \pm 0.01, p<0.001)$ as compared to CONT group. Blocking GABA receptors in $\mathrm{BIC}+\mathrm{SD}$ group increased $I L-6$ expression in non-infarcted area $(2 \pm 0.12$, $p<0.01)$ as compared to SD group.

\section{Effects of acute sleep deprivation on TNF- $\alpha$ expression}

Results of RT PCR studies revealed that the TNF- $\alpha$ expression in non-infarcted area was increased in BIC group $(16.43 \pm 1.15, p<0.001)$ as compared to CONT group. Acute sleep deprivation in SD group reduced TNF- $\alpha$ expression in non-infarcted area $(0.05 \pm 0.009, p<0.001)$ as compared to CONT group and bicuculline administration prior to acute sleep deprivation induction in $\mathrm{BIC}+\mathrm{SD}$ group declined TNF- $\alpha$ expression in non-infarcted area $(0.91 \pm 0.1$, $p<0.001)$ as compared to SD group. TNF- $\alpha$ mRNA expression in infarcted area following blocking GABA receptors in BIC group slightly decreased $(0.9 \pm 0.17)$ and induction of acute sleep deprivation in SD group declined $(0.1$ $\pm 0.02, p<0.001)$ as compared to CONT group. Blocking GABA receptors prior to inducing acute sleep deprivation in $\mathrm{BIC}+\mathrm{SD}$ group increased TNF- $\alpha$ expression in infarcted

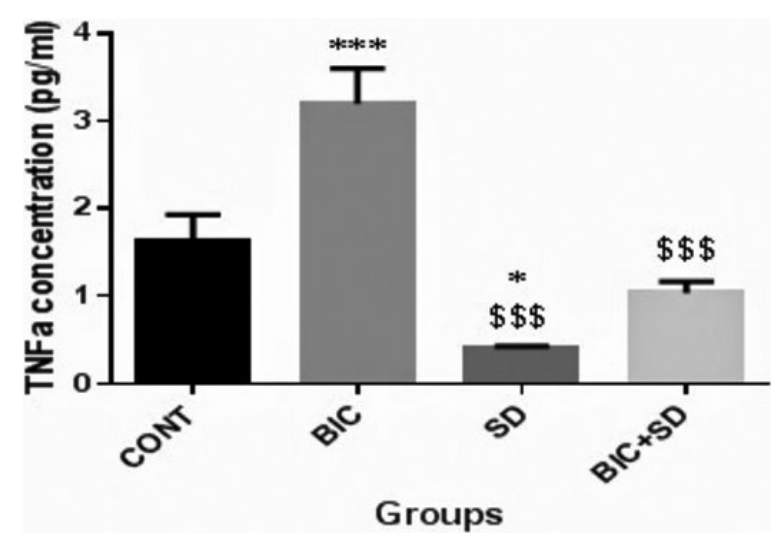

Figure 6. Concentration of TNF- $\alpha$ in serum. All samples were analyzed in duplicate. Data are presented as mean \pm SEM (animals/ group $n=6) .{ }^{*} p<0.05$ and ${ }^{* * *} p<0.001$ vs. CONT group, ${ }^{\$ \$} p<$ 0.001 vs. BIC group. For more abbreviations, see Fig. 2. area $(0.29 \pm 0.18, p<0.001) T N F-\alpha$ expression as compared to $\mathrm{SD}$ group (Fig. $5 \mathrm{C}$ ).

\section{Effects of acute sleep deprivation on TNF- $\alpha$ level in serum}

It is illustrated in Fig. 6 that bicuculline administration in BIC group increased TNF- $\alpha$ level $(3.2 \pm 0.4,1.62 \pm 0.31, p<$ $0.001)$ as compared to CONT group. Acute sleep deprivation in SD group decreased TNF- $\alpha$ level $(0.399 \pm 0.03, p<$ 0.05 ) as compared to CONT group. Bicuculline injection prior to acute sleep deprivation induction in $\mathrm{BIC}+\mathrm{SD}$ group increased $T N F-\alpha$ in serum not significantly as compared to SD group (1.04 \pm 0.13$)$.

\section{Discussion}

In this study, for the first time to our knowledge, we evaluated the effects of acute sleep deprivation on inflammation induced following myocardial ischemia/reperfusion. To assess the myocardial injury, LDH was measured and our results revealed that acute sleep deprivation decreased $\mathrm{LDH}$ levels in serum and showed cardiac protective effect against IR injury. Blocking GABA-A receptors increased this marker of myocardial injury and administration of bicuculline prior to SD attenuated the protective effects of SD. In this regard, Erikson et al. (2017) demonstrated a considerable rise in LDH levels following myocardial IR, however these levels were lower as compared to chronic sleep deprivation (Periasamy et al. 2015; Erikson et al. 2017). In the current study, the myocardial hemodynamic parameters were studied to reflect cardiac functions. We observed that SD increased left ventricular systolic and end diastolic pressure, while bicuculline administration prior SD induction reduced the beneficial effects of SD in this aspect. GABA is involved in regulating cardiac function and GABAergic projections are extended from central nervous system to NTS, the nucleus which has been shown to modulate cardiac performance. GABA receptors are up-regulated during wakefulness which positively modulate the endogenous agonist effects and enhance GABAergic currents (Matsuki et al. 2015), Furthermore, promotes GABA stabilizing and regulatory roles for hemodynamic parameters of the heart (Ma et al. 2015; Hussain et al. 2016; Sallam et al. 2016). Since we aimed to evaluate possible effects of SD in remote areas along with infarcted region, mRNA expression for Hsp70, IL-6 and TNF- $\alpha$ expression was investigated in both infarcted and non-infarcted areas of heart. Our results showed that $H s p 70$ expression in both infarcted and non-infarcted area increased after SD induction and bicuculline administration prior to SD reduced the expression of Hsp70. Hsp70 is known to act as a molecular chaperone to maintain cellular homeostasis and inhibit protein aggregation (Bernardo et al. 2016). It has 
been shown that sleep deprivation for 5 hours produces an elevation of the brain temperature and a significant increase of the muscle tone during the active wakefulness. An increase in brain temperature during sleep deprivation seems to be due to a rise in heat production which would be a stress and lead to activate heat shock proteins (Lapshina et al. 2010). Numerous studies have demonstrated the ability of Hsp70 to attenuate the IR-injury markers of cardiac pathology. Hsp70 has various roles in protein quality control including protein folding/refolding, the ubiquitin proteasome system (UPS) and autophagy. Hsp70 is also known to regulate hypertrophic signaling and the inflammatory response after a cardiac insult (Bernardo et al. 2016). Therefore, up-regulation of $H s p 70$ following SD can be indicative of SD-induced cardioprotection. Pro-inflammatory cytokines contribute to cardiac dysfunction via (i) increasing the generation of toxic oxygen species, (ii) alterations in cardiomyocyte calcium and mitochondrial homeostasis, and (iii) suppression of central sympathetic tone and cardiac baroreflex mechanisms (Sallam et al. 2016). It has been shown that TNF- $\alpha$ and IL- 6 are involved in pathogenesis of cardiovascular diseases (Kleinbongard et al. 2010; Consortium 2012). We found that bicuculline increased the mRNA expression of TNF- $\alpha$ in non-infarcted area and IL- 6 in both infarcted and non-infarcted areas which may suggest bicuculline-created inflammation. Moreover, the elevated IL- 6 can be attributed to the increase in T-cells in the infarcted area through infiltration during reperfusion period (McGinnis et al. 2015). In non-infarcted area, high expression of TNF- $\alpha$ may be the result of activation inflammatory pathways which may induce IL- 6 too. In contrast, SD reduced the expression of these proinflammatory cytokines. In this regard, Irwin and his colleague reported that sleep disturbance can influence the levels of IL- 6 and TNF- $\alpha$ (Irwin et al. 2016), which is difference with our results that could be because of the duration of sleep deprivation.

\section{Conclusion}

GABA shows cardioprotective effects through different mechanisms. In this study, we assessed inflammation as one probable mechanism. Based on our results, TNF- $\alpha$ and IL-6 are down- regulated during acute sleep deprivation and blocking GABA-A receptors in central nucleus of amygdala upregulates these pro-inflammatory cytokines. On the other hand, Hsp70 which has protective effects is upregulated following acute sleep deprivation. Additionally myocardial injury is increased due to bicuculline injection into central nucleus of amygdala.

Acknowledgments. This paper was part of $\mathrm{PhD}$ student thesis project (grant number: 30486 ) and was supported by Tehran University of Medical Sciences.
Conflict of interest. The authors of this study have no conflict of interest.

\section{References}

Abbas A-K (2015): Kainic acid, NMDA and bicuculline induce elevation in concentrations of glutathione and amino acids in vivo: biomarkers for seizure predisposition? J. Behav. Brain Sci. 5, 163-172

https://doi.org/10.4236/jbbs.2015.55017

Arthaud S, Varin C, Gay N, Libourel PA, Chauveau F, Fort P, Luppi PH, Peyron C (2015): Paradoxical (REM) sleep deprivation in mice using the small-platforms-over-water method: polysomnographic analyses and melanin-concentrating hormone and hypocretin/orexin neuronal activation before, during and after deprivation. J. Sleep Res. 24, 309-319 https://doi.org/10.1111/jsr.12269

Avolio E, Mahata SK, Mantuano E, Mele M, Alò R, Facciolo RM, Talani G, Canonaco M (2014): Antihypertensive and neuroprotective effects of catestatin in spontaneously hypertensive rats: interaction with GABAergic transmission in amygdala and brainstem. Neurosci. 270, 48-57

https://doi.org/10.1016/j.neuroscience.2014.04.001

Azizi Y, Faghihi M, Imani A, Roghani M, Nazari A (2013): Postinfarct treatment with [Pyr1]-apelin-13 reduces myocardial damage through reduction of oxidative injury and nitric oxide enhancement in the rat model of myocardial infarction. Peptides 46, 76-82 https://doi.org/10.1016/j.peptides.2013.05.006

Azizi Y, Faghihi M, Imani A, Roghani M, Zekri A, Mobasheri MB, Rastgar T, Moghimian M (2015): Post-infarct treatment with [Pyr 1] apelin-13 improves myocardial function by increasing neovascularization and overexpression of angiogenic growth factors in rats. Eur. J. Pharmacol. 761, 101-108 https://doi.org/10.1016/j.ejphar.2015.04.034

Bernardo BC, Weeks KL, Patterson NL, McMullen JR (2016): HSP70: therapeutic potential in acute and chronic cardiac disease settings. Future Med. Chem. 8, 2177-2183 https://doi.org/10.4155/fmc-2016-0192

Chanana P, Kumar A (2016): GABA-BZD receptor modulating mechanism of Panax quinquefolius against 72-h sleep deprivation induced anxiety like behavior: possible roles of oxidative stress, mitochondrial dysfunction and neuroinflammation. Front. Neurosci. 10, 1-16 https://doi.org/10.3389/fnins.2016.00084

Cheng XY, Gu XY, Gao Q, Zong QF, Li XH, Zhang Y (2016): Effects of dexmedetomidine postconditioning on myocardial ischemia and the role of the PI3K/Akt-dependent signaling pathway in reperfusion injury. Mol. Med. Report. 14, 797-803 https://doi.org/10.3892/mmr.2016.5345

Consortium (2012): The interleukin-6 receptor as a target for prevention of coronary heart disease: a mendelian randomisation analysis. Lancet 379, 1214-1224 https://doi.org/10.1016/S0140-6736(12)60110-X

Dimitrov S, Besedovsky L, Born J, Lange T (2015): Differential acute effects of sleep on spontaneous and stimulated production of tumor necrosis factor in men. Brain. Behav. Immun. 47, 201-210 
https://doi.org/10.1016/j.bbi.2014.11.017

Edalatyzadeh Z, Imani A, Faghihi M, Choopani S, Askari S, Aghajani M, Sadeghniiat-Haghighi K (2016): Effect of acute sleep deprivation on ischemia-induced ventricular arrhythmia in the isolated rat heart. J. Sleep Sci. 1, 38-43

Erikson JM, Valente AJ, Mummidi S, Kandikattu HK, DeMarco VG, Bender SB, Fay WP, Siebenlist U, Chandrasekar B (2017): Targeting TRAF3IP2 by genetic and interventional approaches inhibits ischemia/reperfusion-induced myocardial injury and adverse remodeling. J. Biol. Chem. 292, 2345-2358 https://doi.org/10.1074/jbc.M116.764522

Hussain T, Mir AA, Malik AS, Zahir J, Minhas P (2016): GABAergic premedication and hemodynamic stability during induction laryngoscopy phase of general anesthesia. J. Clin. Med. Res. Updates 3, 17-22 https://doi.org/10.15379/2413-7324.2016.03.04

Imani A, Faghihi M, Sadr SS, Niaraki SS, Alizadeh AM (2011): Noradrenaline protects in vivo rat heart against infarction and ventricular arrhythmias via nitric oxide and reactive oxygen species. J. Surg. Res. 169, 9-15 https://doi.org/10.1016/j.jss.2009.10.025

Irwin MR, Olmstead R, Carroll JE (2016): Sleep disturbance, sleep duration, and inflammation: a systematic review and metaanalysis of cohort studies and experimental sleep deprivation. Biol. Psychiatry 80, 40-52 https://doi.org/10.1016/j.biopsych.2015.05.014

Jeddi S, Asl AN, Asgari A, Ghasemi A (2016): The effect of sleep deprivation on cardiac function and tolerance to ischemiareperfusion injury in male rats. Arq. Bras. Cardiol. 106, 41-48

Kleinbongard P, Heusch G, Schulz R (2010): TNFa in atherosclerosis, myocardial ischemia/reperfusion and heart failure. Pharmacol. Ther. 127, 295-314 https://doi.org/10.1016/j.pharmthera.2010.05.002

Lapshina K, Ekimova I (2010): Study of protective effects of exogenous heat shock protein $70 \mathrm{kDa}$ in model of sleep deprivation in pigeon Columba livia. J. Evol. Biochem. Physiol. 46, 461-470 https://doi.org/10.1134/S0022093010050051

Lavie L, Dyugovskaya L, Golan-Shany O, Lavie P (2010): Heatshock protein 70: expression in monocytes of patients with sleep apnoea and association with oxidative stress and tumour necrosis factor- $\alpha$. J. Sleep Res. 19, 139-147 https://doi.org/10.1111/j.1365-2869.2009.00789.x

Ling H, Gray CB, Zambon AC, Grimm M, Gu Y, Dalton N, Purcell NH, Peterson K, Brown JH (2013): Ca2+/calmodulin-dependent protein kinase ii $\delta$ mediates myocardial ischemia/reperfusion injury through nuclear factor- $\kappa$ B. Circ. Res. 112, 935-944 https://doi.org/10.1161/CIRCRESAHA.112.276915

Lingeshwar P, Kaur G, Singh N, Singh S, Mishra A, Shukla S, Ramakrishna R, Laxman TS, Bhatta RS, Siddiqui HH (2016): A study on the involvement of GABA-transaminase in MCT induced pulmonary hypertension. Pulm. Pharmacol. Ther. 36, 10-21 https://doi.org/10.1016/j.pupt.2015.11.002

Liu X, Yang L, Wellman LL, Tang X, Sanford LD (2009): GABAergic antagonism of the central nucleus of the amygdala attenuates reductions in rapid eye movement sleep after inescapable footshock stress. Sleep 32, 888-896 https://doi.org/10.1093/sleep/32.7.888

Ma P, Li T, Ji F, Wang H, Pang J (2015): Effect of GABA on blood pressure and blood dynamics of anesthetic rats. Int. J. Clin. Exp. Med. 8, 14296-14302

Matsuki T, Takasu M, Hirose Y, Murakoshi N, Sinton C, Motoike T, Yanagisawa M (2015): GABA A receptor-mediated input change on orexin neurons following sleep deprivation in mice. Neurosci. 284, 217-224 https://doi.org/10.1016/j.neuroscience.2014.09.063

McGinnis GR, Ballmann C, Peters B, Nanayakkara G, Roberts M, Amin R, Quindry JC (2015): Interleukin-6 mediates exercise preconditioning against myocardial ischemia reperfusion injury. Am. J. Physiol. Heart Circ. Physiol. 308, 1423-1433 https://doi.org/10.1152/ajpheart.00850.2014

Pace M, Adamantidis A, Facchin L, Bassetti C (2017): Role of REM sleep, melanin concentrating hormone and orexin/hypocretin systems in the sleep deprivation pre-ischemia. PLoS One 12, $1-22$ https://doi.org/10.1371/journal.pone.0168430

Parsa H, Imani A, Faghihi M (2016): Acute sleep deprivation decreases anxiety behavior via GABA-A receptor activation in central nucleus of amygdala. J. Sleep Sci. 1, 139-142

Paxinos G, Watson C (2007): The rat brain in stereotaxic coordinates. 6th ed. Elsevier

Periasamy S, Hsu D-Z, Fu Y-H, Liu M-Y (2015): Sleep deprivationinduced multi-organ injury: role of oxidative stress and inflammation. EXCLI J. 14, 672-683

Rani N, Bharti S, Manchanda M, Nag T, Ray R, Chauhan S, Kumari S, Arya DS (2013): Regulation of heat shock proteins 27 and 70, p-Akt/p-eNOS and MAPKs by Naringin Dampens myocardial injury and dysfunction in vivo after ischemia/reperfusion. PLoS One 8, 1-10 https://doi.org/10.1371/journal.pone.0082577

Riahi E, Khodagholi F, Haghparast A (2013): Role of dorsal hippocampal orexin-1 receptors in associating morphine reward with contextual stimuli. Behav. Pharmacol. 24, 237-248 https://doi.org/10.1097/FBP.0b013e3283635ee9

Sallam MY, El-Gowilly SM, Abdel-Galil A-GA, El-Mas MM (2016): Central GABAA receptors are involved in inflammatory and cardiovascular consequences of endotoxemia in conscious rats. Naunyn Schmiedebergs Arch. Pharmacol. 389, 279-288 https://doi.org/10.1007/s00210-015-1201-7

Winn HR, Welsh JE, Rubio R, Berne RM (1980): Changes in brain adenosine during bicuculline-induced seizures in rats. Effects of hypoxia and altered systemic blood pressure. Circ. Res. 47, 568-577 https://doi.org/10.1161/01.RES.47.4.568

Zagaar MA, Dao AT, Alhaider IA, Alkadhi KA (2016): Prevention by regular exercise of acute sleep deprivation-induced impairment of late phase LTP and related signaling molecules in the dentate gyrus. Mol. Neurobiol. 53, 2900-2910 https://doi.org/10.1007/s12035-015-9176-4

Received: July 11, 2017

Final version accepted: October 9, 2017 